Vol. LXVI 2015

\title{
SETTING-UP OF A CUTTING FORCES MEASUREMENT SYSTEM
}

\author{
TURC Cristian-Gheorghe \\ Faculty of Mechanical Engineering/Department of Materials and Manufacturing Engineering, Politehnica \\ University of Timișoara, Romania, cristian.turc@upt.ro
}

BELGIU George

Faculty of Management in Production and Transportation/Department of Management, Politehnica University of Timișoara, Romania,george.belgiu@upt.ro

BANCIU Felicia Veronica

Faculty of Mechanical Engineering/Department of Materials and Manufacturing Engineering, Politehnica University of Timișoara, Romania, felicia.banciu@upt.ro

\begin{abstract}
The paper is focused in the field of cutting forces measurement by modern sensors and data acquisition systems. It is presented the measurement chain with its components. Thus, there are presented the piezoelectric sensors that are commonly used in modern cutting forces dynamometers construction, as well as some typical topologies. The measurement system includes a data acquisition system that allows the real time data acquisition during the cutting process. The proposed cutting force measurement system can be used in the measurement of three orthogonal forces in milling processes, as well as the measurement of the torque in drilling processes.
\end{abstract}

Key words: cutting force, measurement, sensor, data acquisition

\section{Introduction}

The measurement of cutting forces is critical to the design of cutting tools and machine-tools. Also, the measurement of cutting forces allows:

- The analyse of the cutting process;

- The optimization of the cutting process;

- The designing of better tools.

The knowledge of the cutting forces is very important in the field of the cutting processes optimization. The cutting forces analyse before the beginning of production leads to the increasing of the process capability, and consequently to the increasing of process productivity. Also, the detecting of overloads, collisions and tool breaking could be monitoring using cutting force sensors.

The cutting forces could by measured in proper conditions of precision and technological system integrity only if appropriate sensors are used.

\section{The cutting forces measurement chain}

Figure 1 presents the measurement chain for the measurement of cutting forces. The sensor, which is the heart of a dynamometer, generates a proportional signal to the measured force. This signal is sent through the cable to the amplifier, which is located outside of the machine-tool working area.

\section{Piezoelectric sensors}

The piezoelectric materials (the prefix piezo- comes from the Greek $\pi \imath \varepsilon \varepsilon \xi \varepsilon v$, which means to squeeze or press), generates an electrical charge that is proportional to the applied mechanical load. The charge is developed on the surfaces where the force is applied. Most piezoelectric sensors consist of quartz crystals or piezoceramic elements and work by longitudinal or shear effect.

In case of longitudinal effect sensors (Figure 2), the charge depends only on the applied force $F_{x}$ but not on the dimensions of the crystal disks. The only way to increase the generated charge is to connect several disks (mechanically in series and electrically in parallel). The magnitude of the output charge is:

$$
Q_{x}=d_{11} \cdot F_{x} \cdot n \text {, }
$$


where: $d_{11}$ : the piezoelectric coefficient ( $-2.3 \mathrm{pC} / \mathrm{N}$ for quartz crystals)

$F_{x}$ : the applied force in x-direction

$n$ : number of crystal disks

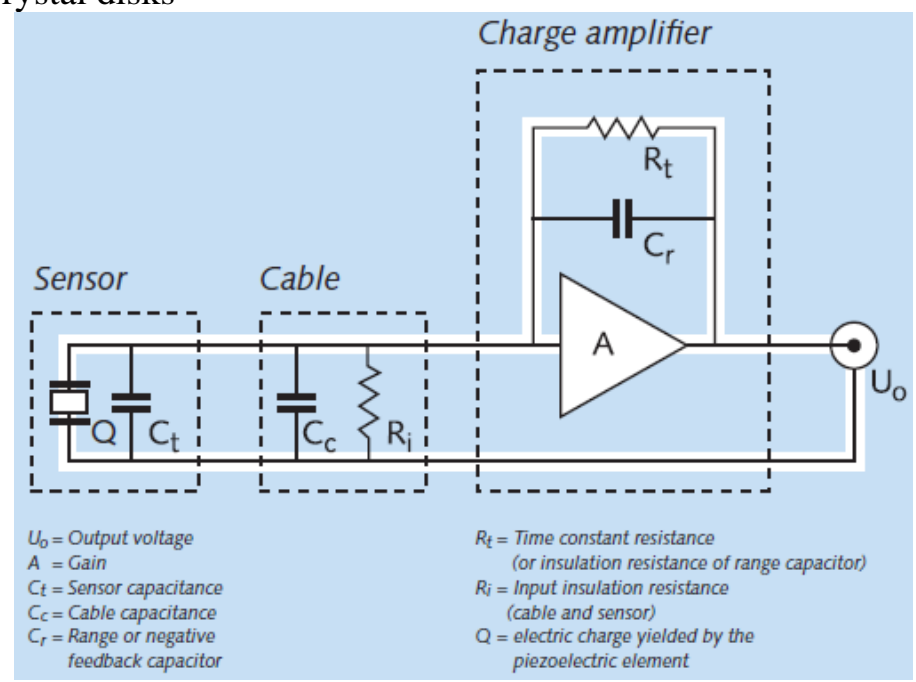

Figure 1: The cutting forces measuring chain

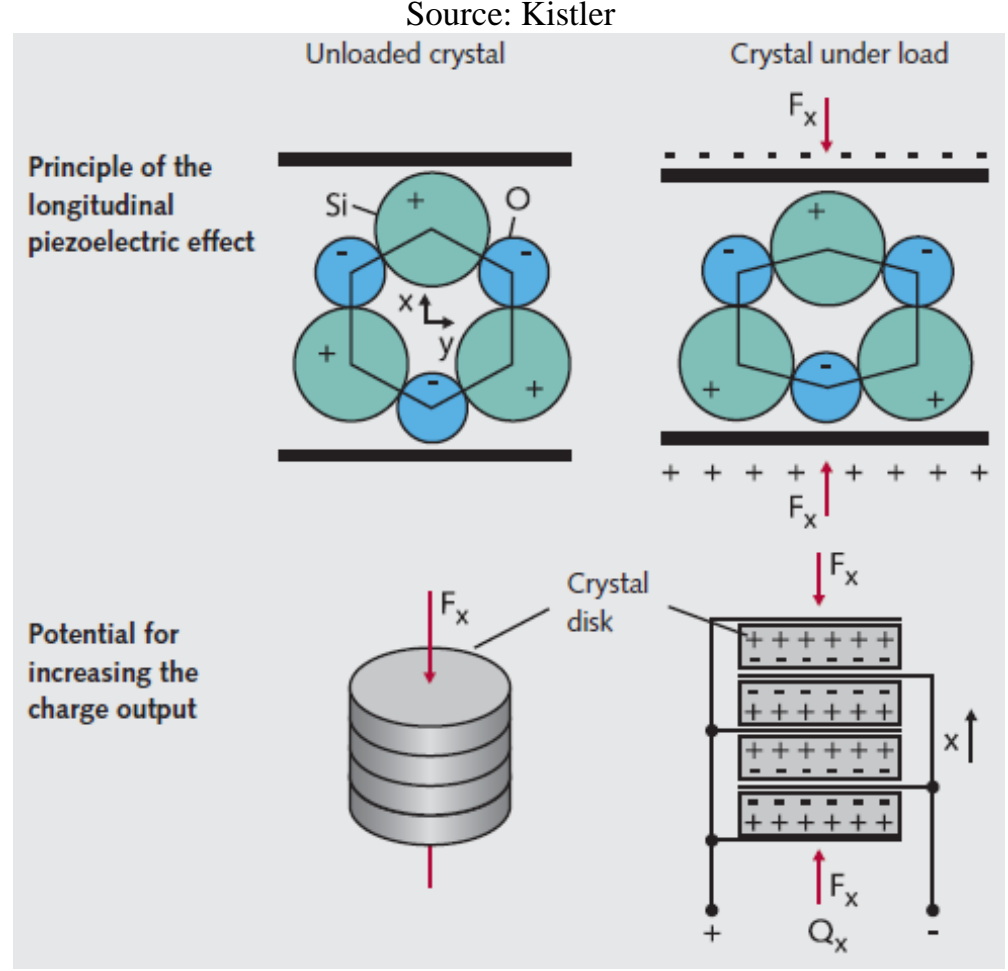

Figure 2: The longitudinal effect sensor

Source: Kistler

The piezoelectric coefficient $d_{11}$ depends on direction and indicates the crystal's degree of force sensitivity in the direction of the corresponding axis.

Also, in case of shear effect sensors (Figure 3), the charge depends only on the applied load but not on the dimensions of the crystal disks. The magnitude of the output charge is:

$$
Q_{x}=2 \cdot d_{11} \cdot F_{x} \cdot n
$$

\section{Dynamometers based on piezoelectric sensors}

Piezoelectric based dynamometers are very compact/rigid systems, so they have a high natural frequency that allows precise measurement of highly dynamic processes. The rust-resistant design and the protection against ingress of cutting fluid achieve an IP67 rating. The quality of these measurement systems ensures a very long - almost unlimited - service life.

Figure 4 shows two typical topologies of cutting forces dynamometers. 


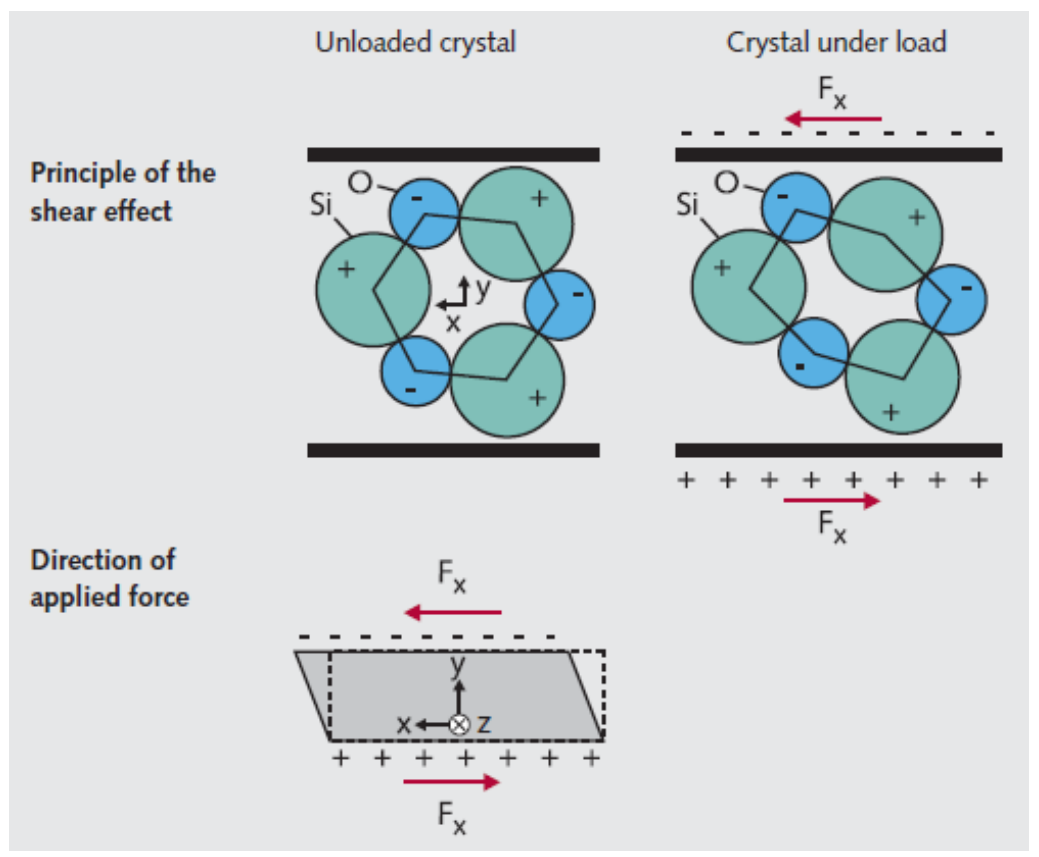

Figure 3: The shear effect sensor

Source: Kistler

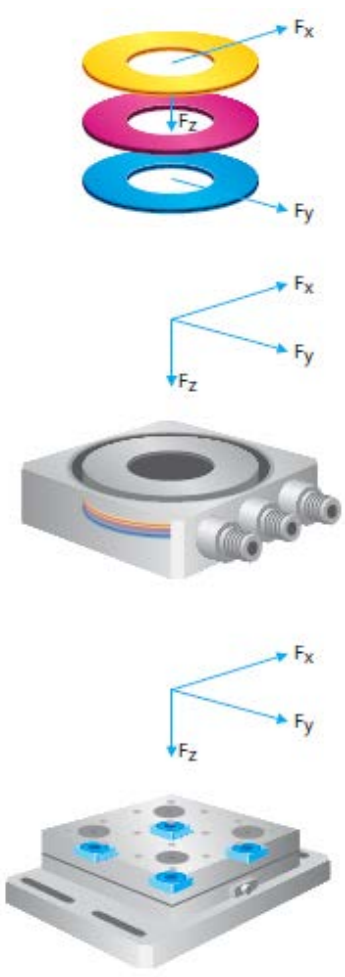

a) Dynamometer for 3 components: $F_{x}, F_{y}, F_{z}$
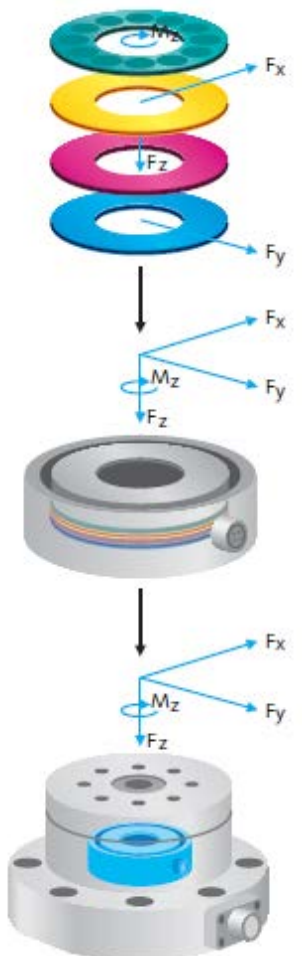

b) Dynamometer for 4 components:

$$
F_{x}, F_{y}, F_{z}, M_{z}
$$

Figure 4: The construction of cutting forces dynamometers Source: Kistler

A 3-component sensor consists of two shear quartz - for $F_{x}$ and $F_{y}$ - and one pressure quartz for $F_{z}$ - that are incorporated in a single case. Four of these 3-component sensors are installed between a base plate and a top plate under high preload. The outputs of the four built-in force sensors are interconnected in the dynamometer in such a manner that multi-component force and moment measurements are also possible. 
A 4-component sensor consists of two shear quartz - for $F_{x}$ and $F_{y}$, one pressure quartz - for $F_{z}$, and one special sensor - for $M_{z}$, which uses shear-sensitive plates that are arranged in a circle so that their shear-sensitive axes are tangential.

\section{The charge amplifier}

The charge amplifier (Figure 1) converts the charge produced by a piezoelectric sensor into a proportional voltage that is used as an input variable for monitoring and control processes. A charge amplifier basically consists of an inverting voltage amplifier with high open-loop gain and capacitive negative feedback. Multi-component dynamometers require a corresponding number of measuring channels, i.e. a corresponding number of charge amplifiers.

\section{The realized measurement system}

Figure 5 shows a general view of the realised cutting forces measurement system. It consists of:

- Kistler 9272, a 4-component dynamometer (Figure 6)

- Kistler charge amplifier ICAM 5073A411 (Figure 7)

- Connecting system (cables and distribution box - Figure 8)

- Signal conditioning and data acquisition system (Figure 9)

- PC with data acquisition and calibrating software

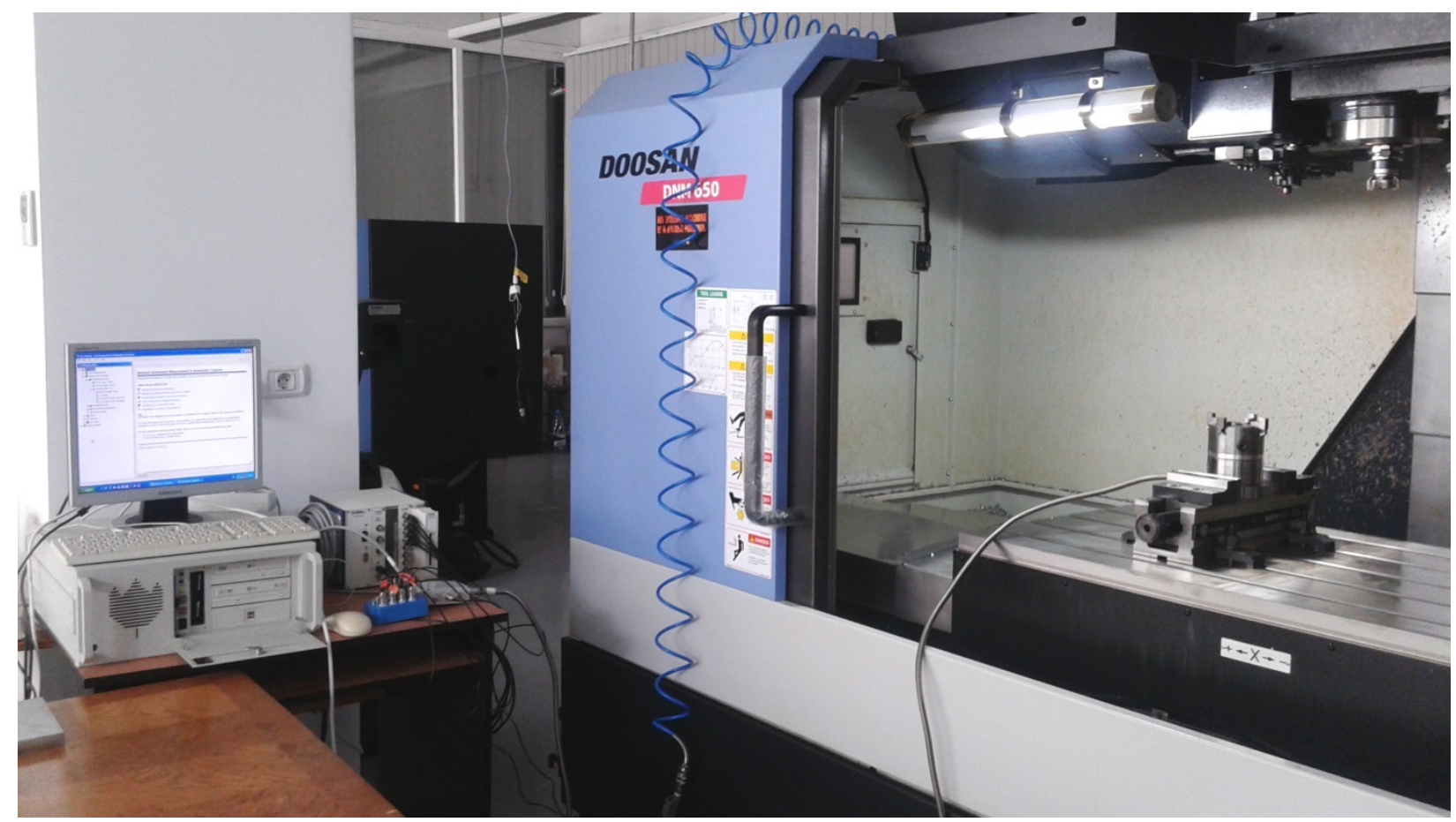

Figure 5: The realised cutting forces measurement system

The 4-component dynamometer allows the measurement of three orthogonal forces in milling processes, as well as the measurement of the torque in drilling processes. The dynamometer is equipped by a four jaw chuck in order to quickly install the work piece. An intermediary piece is needed in order to mount the chuck on the upper face of the dynamometer. This construction allows the installation of circular (Figure 6.a) or rectangular pieces (Figure 6.b). The whole system is installed on the machine-tool table through a machine vice.

The charge amplifier ICAM 5073A411 is a 4-channel industrial charge amplifier that allows the converting of each charges from the four sensors of the dynamometer to proportional voltages. Each channel of the amplifier must be calibrated according to the dynamometer sensors characteristics, in order to achieve precise conversions. The calibration is performed by the PC, through the RS-232 serial interface and calibrating software.

The four voltage signals are sent to the signal conditioning system, which is a National Instruments SCXI based system. It consists of a chassis that is equipped by data acquisition and signal conditioning modules. 


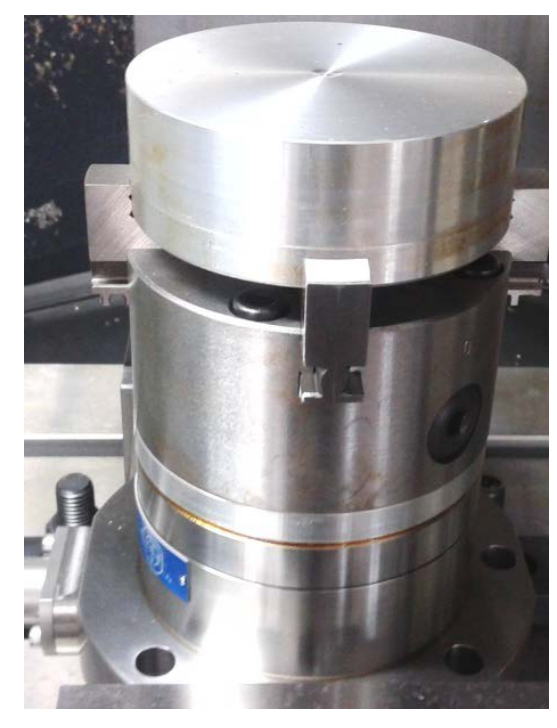

a) Holding of circular work pieces

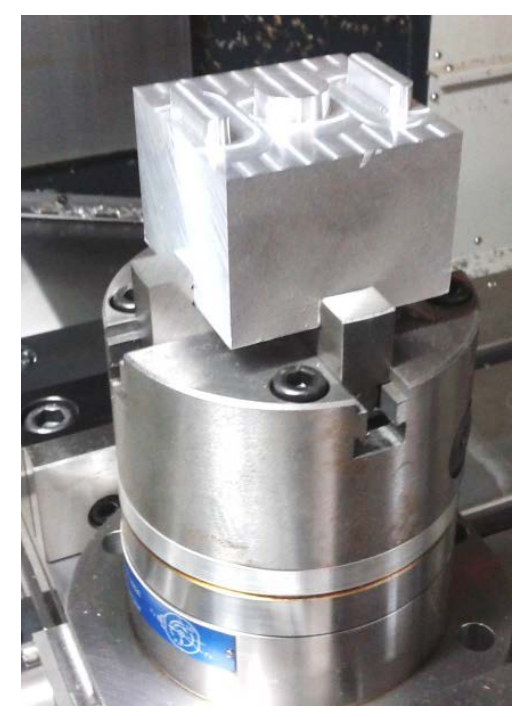

b) Holding of rectangular work pieces

Figure 6: The 4-component dynamometer Kistler 9272

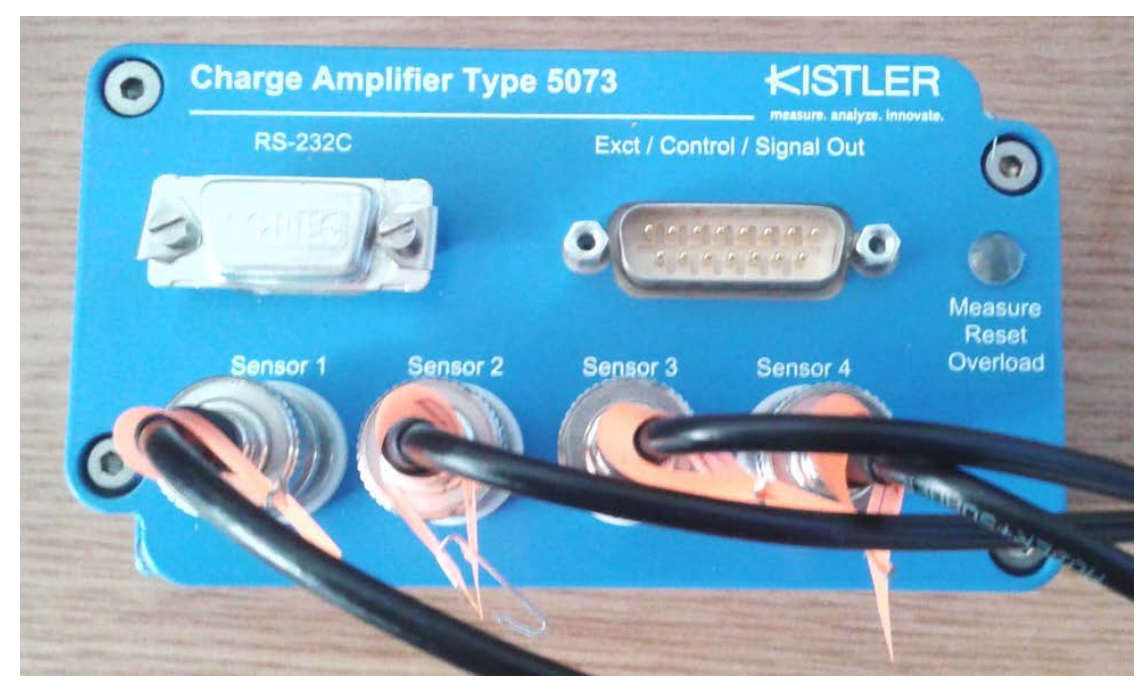

Figure 7: The charge amplifier Kistler 5073A411

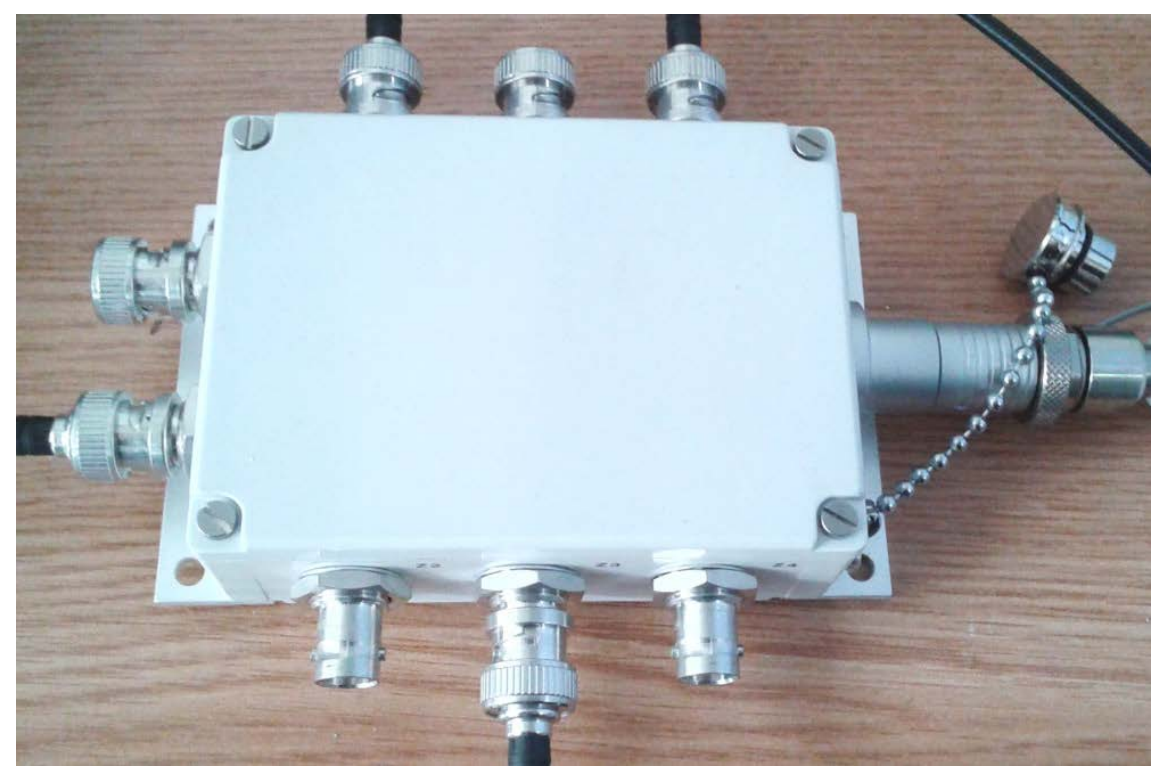

Figure 8: The distribution box 


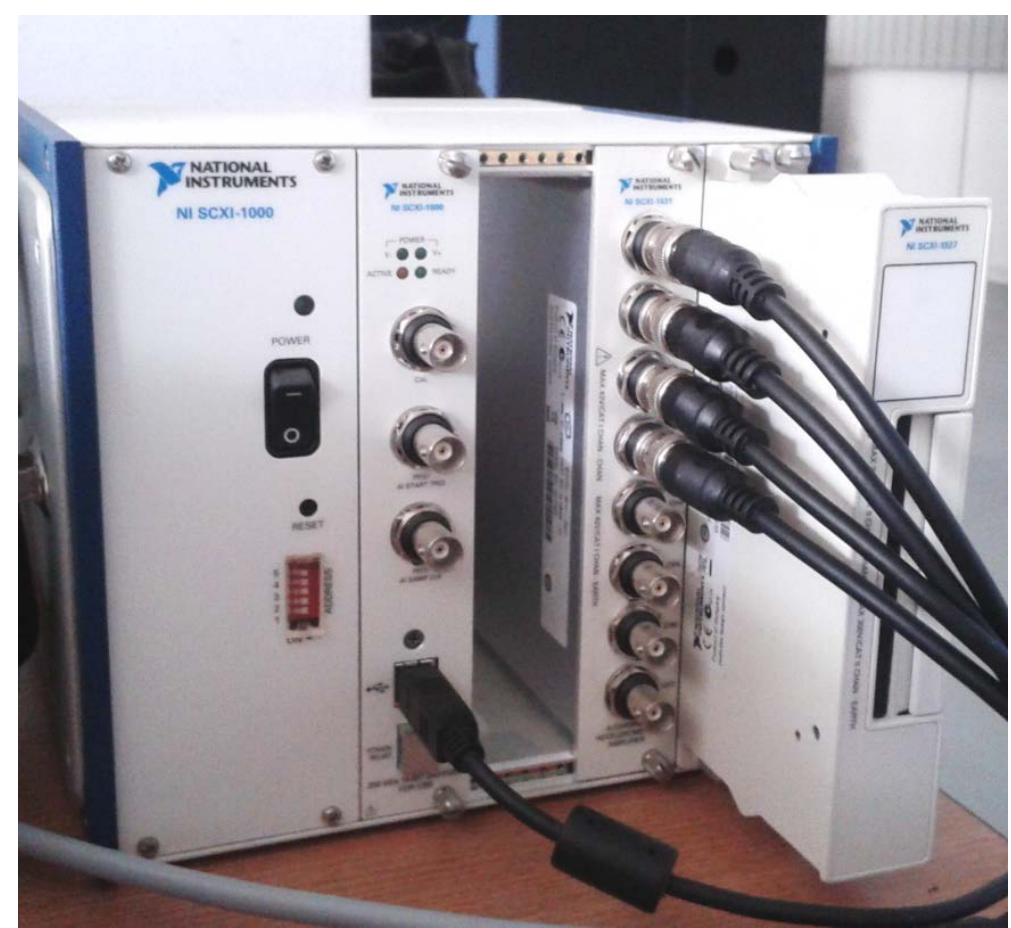

Figure 8: The signal conditioning and data acquisition system

\section{Conclusions}

The presented cutting forces measurement system is a reliable research tool. It can be used in monitoring of milling or drilling processes. The core of the system is a 4-component dynamometer based on piezoelectric sensors technology.

\section{References}

- Wilson, Jon. et. al. Test and Measurement, Elsevier Inc., (2009).

- $\quad * * *$, Cutting Force Measurement, Kistler Group.

- $\quad * * *$, 4-Component Dynamometer, Kistler Group.

- $\quad$ ***, Industrial Charge Amplifier for Applications in Manufacturing, Kistler Group.

- $\quad * * *$, http://www.ni.com 